\title{
Effect of Strategy-Homogeneity on the Prisoner's Dilemma Game in a Square Lattice
}

\author{
Mengqin Yang1, Hedong $\mathrm{Xu}^{2}$, Cong $\mathrm{Li}^{3}$, Suohai Fan ${ }^{1}$ \\ ${ }^{1}$ School of Information Science and Technology, Jinan University, Guangzhou, China \\ ${ }^{2}$ Shenzhen Stock Exchange, Postdoctoral Programme, Shenzhen, China \\ ${ }^{3}$ School of Systems Science and Engineering, Sun Yat-sen University, Guangzhou, China \\ Email: mengqinyoung@163.com
}

How to cite this paper: Yang, M.Q., Xu, H.D., Li, C. and Fan, S.H. (2021) Effect of Strategy-Homogeneity on the Prisoner's Dilemma Game in a Square Lattice. Journal of Applied Mathematics and Physics, 9, 295-305. https://doi.org/10.4236/jamp.2021.92021

Received: January 25, 2021

Accepted: February 22, 2021

Published: February 25, 2021

Copyright $\odot 2021$ by author(s) and Scientific Research Publishing Inc. This work is licensed under the Creative Commons Attribution International License (CC BY 4.0).

http://creativecommons.org/licenses/by/4.0/

\begin{abstract}
We investigate the effect of strategy-homogeneity on the prisoner's dilemma game in a square lattice. Strategy-homogeneity means that the population contains at least one connected group in which individuals maintain the same strategy at each iteration and may update according to updating rule at next iteration. The simulation results show that the introduction of strategy-homogeneity increases the cooperation in the evolutionary stable state. For any value of temptation to defect, the density of cooperators in equilibrium state increases firstly and then decreases as the level of strategy-homogeneity increases constantly, and there exists an appropriate level of strategy-homogeneity, maximizing the density of cooperators. The results may be favorable for comprehending cooperative behaviors in societies composed of connected groups with coherent strategy.
\end{abstract}

\section{Keywords}

Strategy-Homogeneity, Prisoner's Dilemma Game, Square Lattice, Cooperative Behavior

\section{Introduction}

Cooperative behaviors appear widely from biology to social systems [1]-[10] However, cooperation means sacrificing personal interest for the collective benefits when a defector obtains more from his cooperative opponent than a cooperator does. Therefore, it is vital to investigate why individuals choose to cooperate with others. The classical game theory is firstly proposed by Nash [11], forming a research paradigm framework based on classical game. In this theory, all of players are assumed to be of perfect rationality [11], indicating that they always make the best decisions. In practice, people are bounded rational in 
realistic world [12] [13] as a result of being influenced by others. In view of this, evolutionary game theory [14] [15] is put forward, aiming at studying about the evolutionary process of cooperation within a population on social dilemma [16] [17]. In evolutionary game theory, payoff is represented by fitness, and strategies are changing over iteration constantly until equilibrium state is reached. Subsequently, Axelrod and Hamilton [18] investigate the evolutional course of cooperation by considering repeated prisoner dilemma. Nowak and May combine spatial structure with evolutional classical game [19] [20] creatively, generating the networked evolutionary game. Above all, network evolutionary game provides a powerful framework for the research of the cooperation.

It is a matter of fact that societies, in almost every age and region, always contain at least one connected group in which individuals maintain the same strategy at each iteration and may update according to updating rule at next iteration. This is the strategy-homogeneity we will investigate in this paper. This idea comes from the following related pieces of literature, namely, Ref. [21] [22], studying strategy-assortativity previously, but the strategy-assortativity referred by them is used to describe people's general trend to interact with those who act like them, leading to the formation of relations. More specifically, in Ref. [21], an individual selects his partner from the assortative pool with a fixed probability that represents strategy-assortativity or from the random pool with the corresponding probability. In Ref. [22], the strategy-assortativity reflected by the fixed probability decides what kind of investors' partner in the game.

The strategy-homogeneity is equivalent to the initial strategy distribution, but the literature focused on the impact of initial strategy distribution on the evolution of cooperation for social dilemmas has so far given little attention to the influence of the level of strategy-homogeneity in the network. A part of the existing literature on initial strategy distribution is shown in the following. Szabó and Fáth unveil the fact that the result of evolutionary games is closely related to initial conditions [23]. For instance, in Ref. [24], the authors investigate not only the effect of different initial distribution of defectors on cooperators under the premise of same initial frequency of defectors but also the influence of dynamic initial frequency of defectors on cooperators under the same initial distribution of defectors. They found that the situation where defectors are located on lowest-degree vertices initially can display more robust cooperation than other situations. Then, the initial configuration that all $S \times S$ individuals are defectors except for an $s \times s(s=1,3,5, \ldots, 15$ and 30) cluster of cooperators in the center of the lattice is investigated, and their simulations confirm that the probability of invasion is essentially independent of the initial number of cooperators provided that they form at least a $3 \times 3$ cluster [25]. By assuming a small fraction of zealous cooperators, Masuda shows that a large fraction of cooperation emerges in evolutionary dynamics of social dilemma games [26]. Other related studies on initial configurations can be seen in literature [27] [28] [29] [30].

In general, it is worthy of special consideration to investigate the effect of strategy-homogeneity on individual's behaviors. Therefore, this paper will focus 
on the effect of strategy-homogeneity on the prisoner's dilemma game in a square lattice.

The structure of the paper is as follows. Section 2 elaborates the strategy-homogeneity and corresponding strategy update rule. Section 3 shows the simulation results. Section 4 concludes.

\section{Strategy-Homogeneity and Corresponding Strategy Update Rule}

Strategy-homogeneity means that the population contains at least one connected group in which individuals maintain the same strategy at each iteration and may update according to updating rule at next iteration. For example, various connected groups are shown in bold black in Figure 1. We use strategy-homogeneity $p$, namely, the level of strategy-homogeneity, as an index to form different connected groups here. In addition, the strategy-homogenous edge is defined as this kind of edge through which individuals keep same strategy at each iteration and may update according to updating rule at next iteration.

Before the idea of strategy-homogeneity is realized, we need know how to select strategy-homogenous edge according to strategy-homogeneity $p$ and divide all agents into various groups. Firstly, given the strategy-homogeneity $p$, we will compare a random number generated for each edge with $p$, and the corresponding edge is then selected if the random number is less than $p$. Strategy-homogeneity $p$ is about the proportion of strategy-homogenous edges. Secondly, nodes involved by connected strategy-homogenous edges constitute a group. Moreover, a single node that is not involved by any strategy-homogenous edge constitutes a group itself. So, the population is divided into various connected subgraphs in which the number of individuals may be 2,3 and so on. Taking the strategy-homogenous edge $X Z$ depicted in Figure 1 for example, the nodes $X$ and $Z$ constitute one connected group, while the single node such as $Y$ constitutes another group.

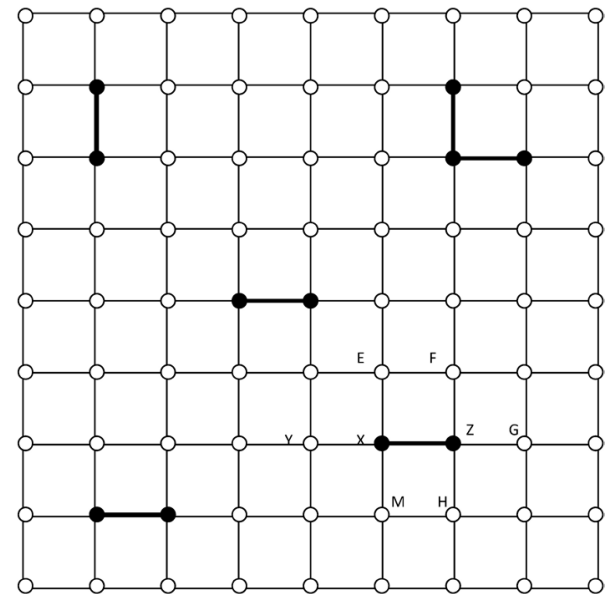

Figure 1. Graphic presentation and brief description about strategy-homogeneity in a square lattice are provided. The level of strategy-homogeneity is set to 0.05 and various groups are shown in this figure. 
Individuals acquire their accumulated payoffs from games with their nearest neighbors. For a group, suppose the node $X$ obtains maximum payoff $P(X)$ within this group, and the node $Y$ randomly selected from all neighbors of this group gains payoff $P(Y)$. All individuals in this group maintain consistent strategy at each iteration, thus $X$ and all individuals in this group will adopt the strategy of $Y$ through Fermi updating rule with probability as follows:

$$
W(Y \rightarrow X)=\frac{1}{1+\exp [(P(X)-P(Y)) / \kappa]}
$$

$\kappa$ characterizes the noise effects, including fluctuations in payoffs, individual trials and errors in decision. We set $\kappa=0.1$.

All individuals in any one group should be assigned the consistent strategy initially. It should be noted that there are no strategy-homogenous edges in the population when $p=0$. And $p$ value is in the range of $[0,0.45]$. If $p$ value is too big, it is more likely that all individuals are either cooperators or defectors. In next section, we present the simulation results in square lattice and get clear on the effect of strategy-homogeneity on the evolutionary cooperative behavior.

\section{Simulation Results in Square Lattice}

The model we use in this paper is a two-player non-cooperative game called the Prisoner's Dilemma (PDG), firstly described in [31]. The PDG is considered a vital model for studying the emergence of cooperation among selfish individuals [14] [32] [33]. In the traditional PDG, the reward for mutual cooperation is $R$, the punishment for mutual defection is $P$, and the mixed strategies give the cooperator $S$ and the defector $T$ respectively. The dilemma holds the inequation $T>R>P>S$ and constraint $T+S<2 R$. Without loss of generality, we consider $R=1, T=b, P=S=0$, and $b$ ranges in $(1,2]$, as proposed by Nowak and May in [19] [20].

Simulation results are obtained from Monte Carlo simulation in square lattice with a system size of $100 \times 100$ through varying $b$ and $p$. Strategies of groups mentioned in the model are randomly selected from cooperation and defection with the probability 0.5 initially. Players update their strategies synchronously during each iteration. To overcome the influence of randomness, the end results are averaged with 40 independent simulation each of which contains 10,000 iterations. Each data in the equilibrium state is gained through averaging the last 1000 steps.

Figure 2(a) presents the density of cooperators as a function of iteration for different probability $p$ values range in $[0,0.45]$. In general, the density of cooperators increases first and then decreases in equilibrium state as $p$ increases constantly. In detail, in equilibrium state, the density of cooperators keeps increasing when $p$ increases from 0 to 0.35 , and the density of cooperators then keeps decreasing when $p$ increases from 0.35 to 0.45 . Namely, the density of cooperators gets its maximum at $p=0.35$. It is consistent with the literature of the classic 
prisoner's dilemma game in [34] [35] for the result of $p=0$, and the introduction of $p$ increases the cooperation in the evolutionary stable state. Here, for $p \geq$ 0.2 , the proportion of cooperators in equilibrium state is higher than the initial state. It means that there is an increase in the number of cooperators, leading to a stable state in which cooperators dominate.

Considering the effect of $p$ on cooperation under more $b$ values, we explore cooperative level in dependence on the temptation to defect $b$ for various $p$ values, as shown in Figure 2(b). For a fixed $p$, the density of cooperators is a decreasing function of $b$. It is worth noting that with the increase of $b$, especially when $b=1.8$, all cooperators for all $p$ values are annihilated. It is certain that cooperative level for $p=0$ is the lowest than other $p$ values. Both the trend of cooperative level for various $b$ in equilibrium state and $b$ value making cooperation vanish are same with the result of [35] in the circumstance of $p=0$. We can see clearly that there is an optimal $p$ value for any $b$ value. Particularly, for $b=$ 1.1 , the density of cooperators in equilibrium state increases first and then decreases as $p$ increases, which is in accordance with Figure 2(a).

Next, we will explain results in Figure 2(a) from different perspectives. The density of cooperators being learnt in each round is of great significance because all individuals only change their strategy through learning. Frankly, cooperative behavior will be prosperous when the density of cooperators being learnt is high. Seen from Figure 3, in equilibrium state, the density of cooperators and the density of cooperators being learnt have the same trend, namely, a trend of increasing first and then decreasing as $p$ increases constantly. When $p \geq 0.2$, the leading role of the cooperators in equilibrium state is due to the dominant density of cooperators being learnt in the final iteration.

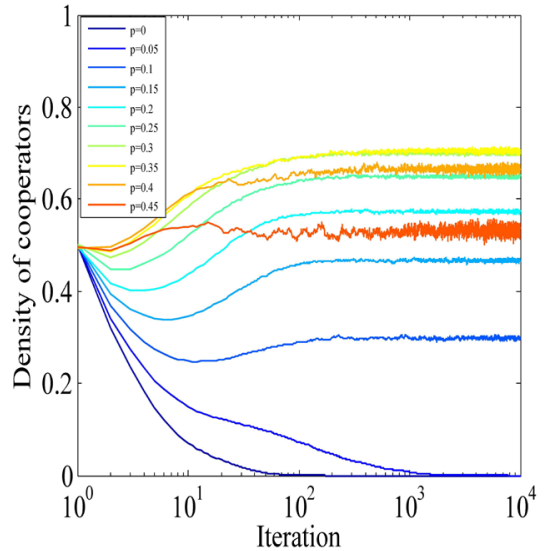

(a)

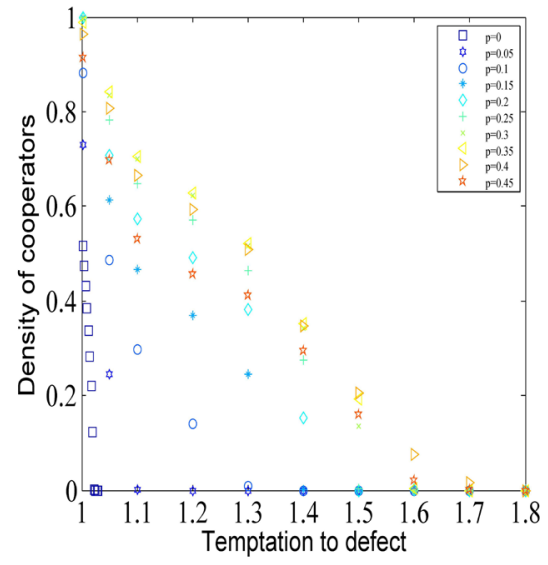

(b)

Figure 2. (a) describes evolutional density of cooperation for fixed $b=1.1$ and different $p$ values range in $[0,0.45]$ where interval is 0.05 . The density of cooperators refers to the proportion of the number of individuals who adopt cooperation to the total number of individuals, with the value between $[0,1]$. (b) describes equilibrium cooperative level as a function of temptation to defect $\mathrm{b}$ with different $p$ values range in $[0,0.45]$ where interval is 0.05 , and the temptation to defect $b$ varies from 1.0025 to 1.8 . 


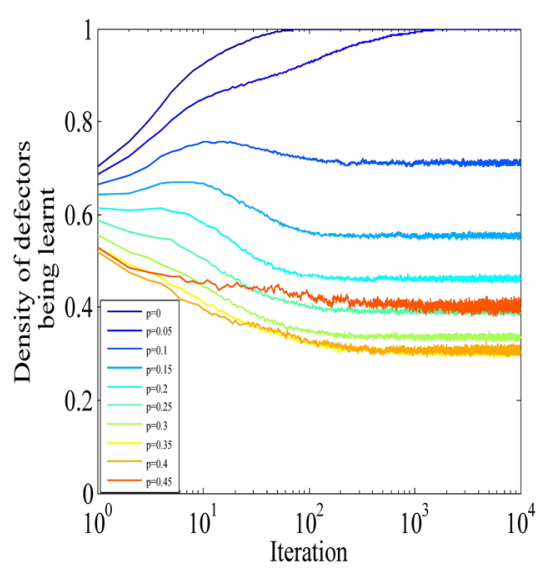

(a)

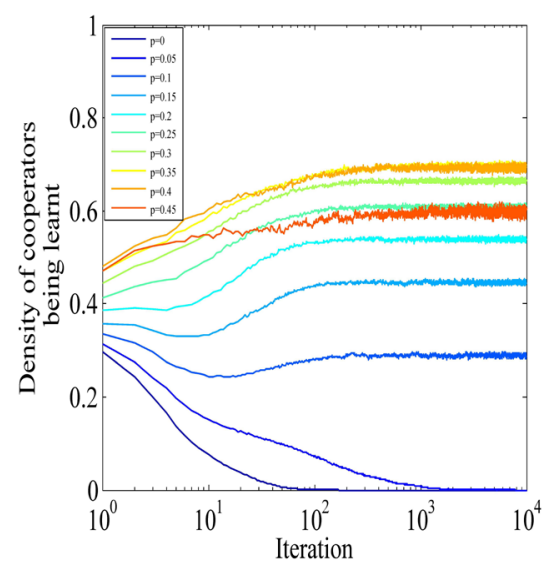

(b)

Figure 3. (a) and (b) describe evolutional density of cooperators being learnt and evolutional density of defectors being learnt respectively in square lattice for same setup as Figure 2(a).

We understand the effect of strategy-homogeneity $p$ on the persistent of cooperative behavior through snapshots of strategic distribution in equilibrium state, as shown in Figure 4. Figure 4(d) shows that a small fraction of cooperators can prevail through forming clusters. When strategy-homogeneity $p$ increases, both the size of clusters and the number of cooperators in Figure 4(h) and Figure 4(1) are bigger than Figure 4(d) at the end of evolution.

Figure 5 shows the equilibrium average payoff of cooperators and defectors respectively for different $p$. We have known that defectors dominant in equilibrium state for $p=0.1$, but cooperators earn more average payoff than defectors under this $p$ value. We infer that the payoff of cooperators may be related to the formation of cooperative clusters. The payoff of defectors increases with the increase of their cooperative neighbors when $p$ increases from 0.1 to 0.3 . As $p$ continues to increase to 0.35 , defectors are hard to invade bigger cooperative clusters, and less cooperators appear in the neighbors of defectors, hence there is a descending trend in defector's payoff. In circumstance of $p=0$ and $p=0.05$, the payoff of all individuals is 0 because there is no cooperator in the population.

Furthermore, as Figure 6 displays, we will use strategic perturbation rate during the process of iteration to measure whether strategies of all individuals remain unchanged or only small perturbations. In Figure 6, strategic perturbation rate is not zero from beginning to end for three $p$ values. Although strategic perturbation rate decreases with the increase of $p$, the range of fluctuation of strategic perturbation rate is indeed increasing. The larger $p$ is, the greater the fluctuation of the number of changes in all individual strategies during whole iteration. In other words, take $p=0.45$ for example, the number of individuals who change strategies varies greatly, which may lead to the failure of equilibrium state and the failure of convergence for cooperative density. It is reasonable that this paper only considers the effect of $p$ in the range of $[0,0.45]$ on the density of cooperators. 


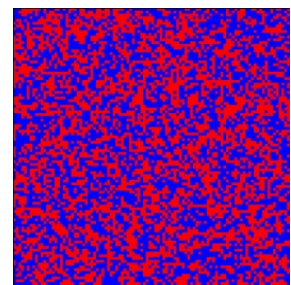

(a) $t=1$

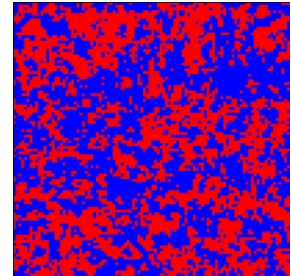

(e) $t=1$

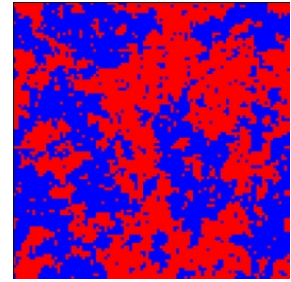

(i) $t=1$

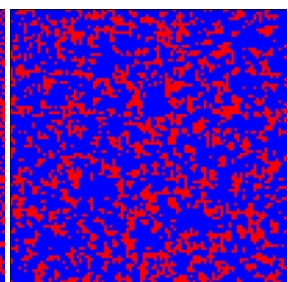

(b) $t=3$

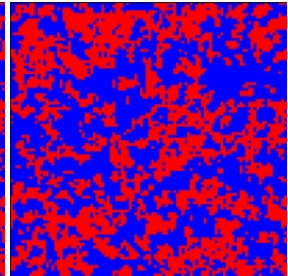

(f) $t=2$

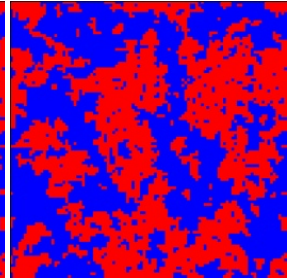

(j) $t=2$

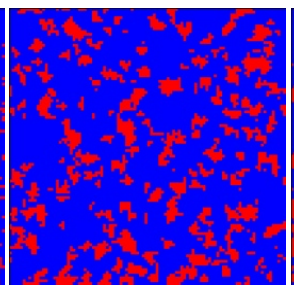

(c) $t=18$

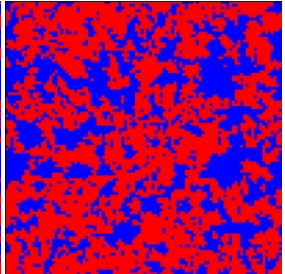

(g) $t=10$

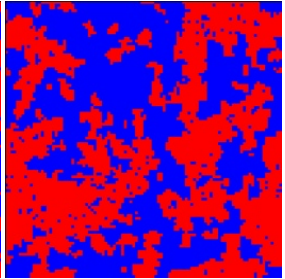

(k) $t=5055$

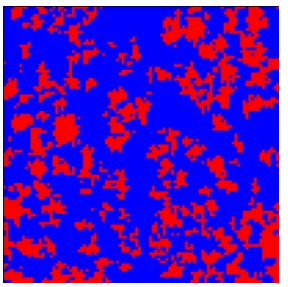

(d) $t=10,000$

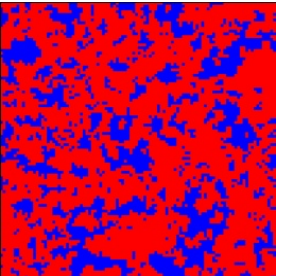

(h) $t=9999$

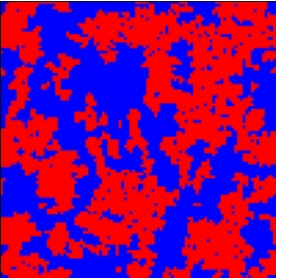

(l) $t=10,000$

Figure 4. The first row, second row and third row represent snapshots of typical distributions for cooperators (red) and defectors (blue) of $p=0.1, p=0.35$ and $p=0.45$ respectively at different time step $t$ in square lattice. The temptation to defect $b=1.1$.

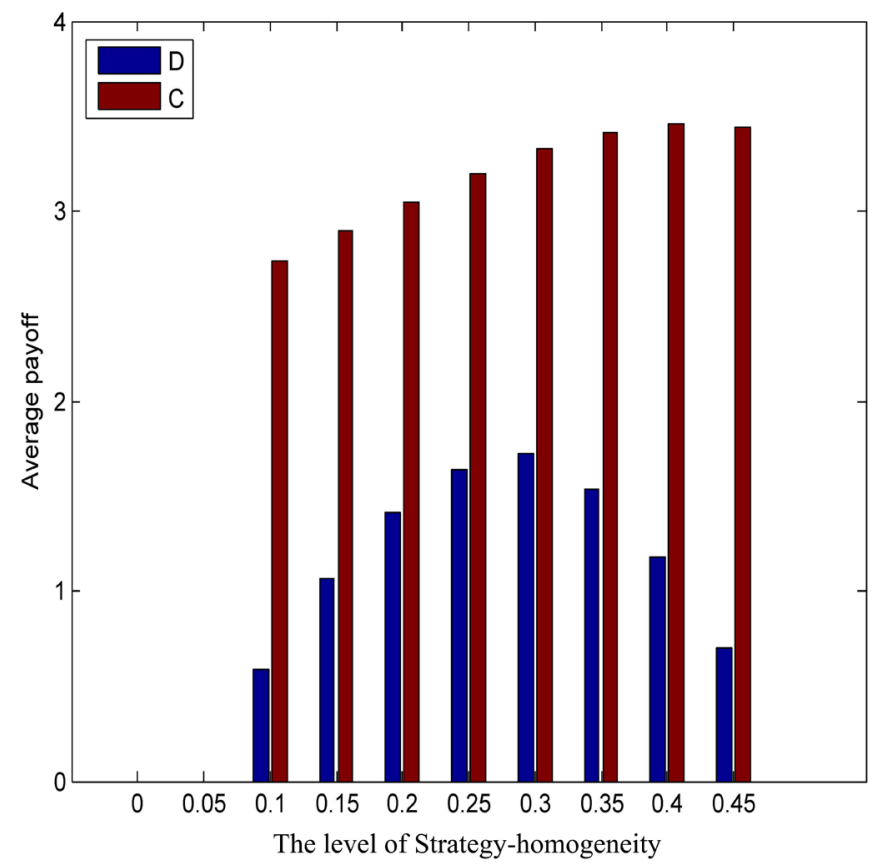

Figure 5. The equilibrium average payoff of cooperators and defectors are shown respectively as a function of the level of strategy-homogeneity $p$ ranges in $[0,0.45]$ where interval is 0.05 . Similarly, we set $b=1.1$. 


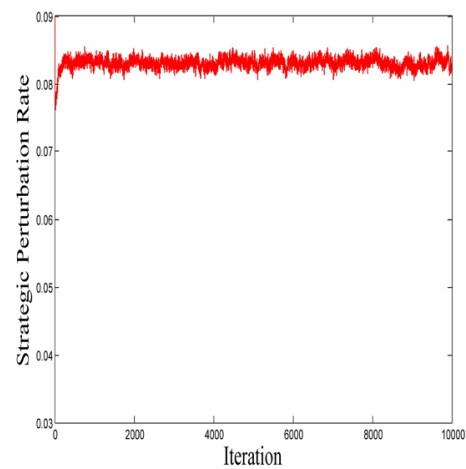

(a)

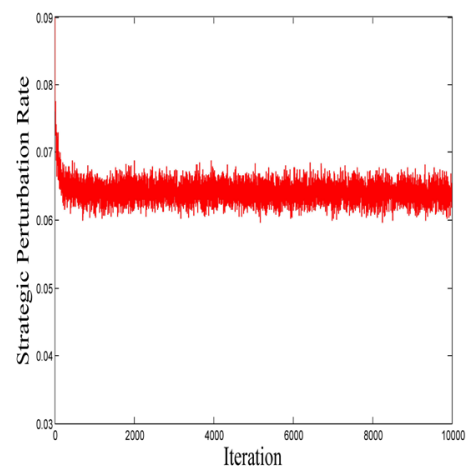

(b)

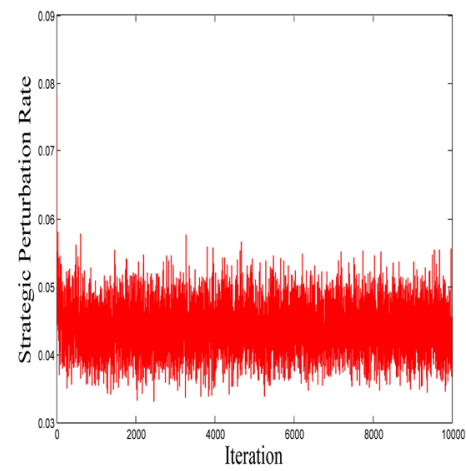

(c)

Figure 6. (a), (b) and (c) represent strategic perturbation rate of evolutionary process for $p=0.1, p=0.35$ and $p=0.45$ respectively. Strategic perturbation rate at time step $t$ is the ratio of total number of variation in all individual's strategies in the process of iteration from t to $t+1$ and the total number of all individuals in square lattice. We set $b=1.1$.

Considering the effect of $p$ on cooperation under more $\kappa$ values, we explore cooperative level in dependence on selection pressure $\kappa$ for various $p$ values, as shown in Figure 7. On the one hand, for any $\kappa$ value, with the increase of $p$, the change trend of cooperative level in equilibrium state is consistent. On the other hand, compared with $p=0$ and $p=0.05$, cooperative level in equilibrium state under other $p$ values are improved at different degree. For example, the cooperative level of $p=0.15$ is higher than that of $p=0$ and $p=0.05$, the cooperative level of $p=0.2$ is also higher than that of $p=0$ and $p=0.05$, but the degree of increase is different. In general, the change of $\kappa$ will not affect the previous conclusion. 


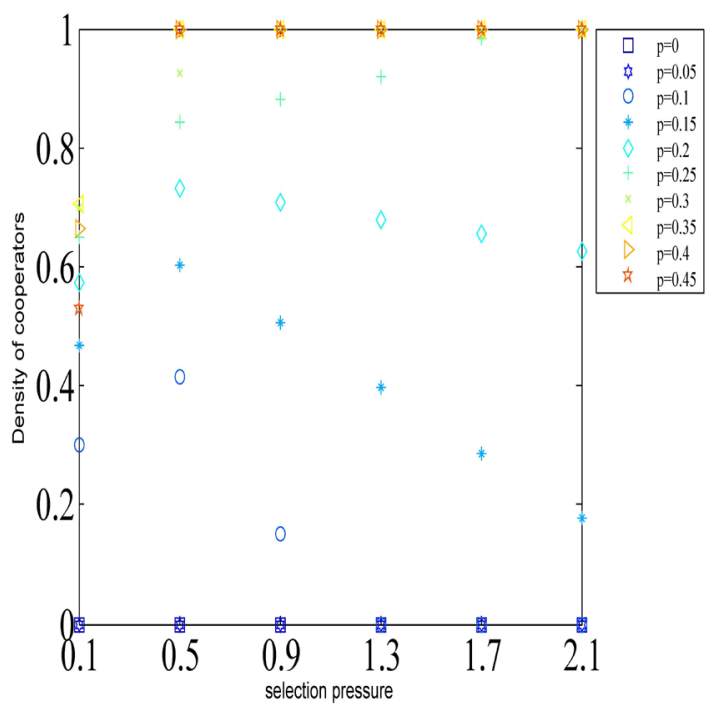

Figure 7. Equilibrium cooperative level as a function of selection pressure $\kappa$ with different $p$ values range in [0,0.45] where interval is 0.05 . The temptation to defect $b$ is 1.1 .

\section{Conclusion}

The strategy-homogeneity in this paper means that the population contains at least one connected group in which individuals maintain the same strategy at each iteration and may update according to updating rule at next iteration. The strategy-homogeneity $p$ is used to control the proportion of strategy-homogenous edges initially, thus affecting the evolution of strategies. We find that the introduction of strategy-homogeneity increases the cooperation in the evolutionary stable state. For any value of temptation to defect, the density of cooperators in equilibrium state increases firstly and then decreases as the level of strategy-homogeneity increases constantly, and there exists an appropriate level of strategy-homogeneity, maximizing the density of cooperators. The results may be favorable for comprehending cooperative behaviors in societies composed of connected groups with coherent strategy.

\section{Acknowledgements}

This work is supported by the National Natural Science Foundation of China (Grant no. 61572233), and the National Social Science Foundation of China (Grant no. 16BTJ032).

\section{Conflicts of Interest}

The authors declare no conflicts of interest regarding the publication of this paper.

\section{References}

[1] Neumann, J.V. and Morgenstern, O. (1953) Theory of Games and Economic Behavior. Princeton University Press, Princeton. 
[2] Dugatkin, L.A. (1997) Cooperation among Animals: an Evolutionary Perspective. Oxford University Press, Oxford.

[3] Sigmund, K. (2010) The Calculus of Selfishness. Princeton University Press, Princeton. https://doi.org/10.1515/9781400832255

[4] Turner, P.E. and Chao, L. (1999) Prisoner's Dilemma in an RNA Virus. Nature, 398, 441-443. https://doi.org/10.1038/18913

[5] Johnson, N. and Lux, T. (2011) Financial Systems: Ecology and Economics. Nature, 469, 302-303. https://doi.org/10.1038/469302a

[6] Wang, X.W., Jiang, L.L. and Wang, B.H. (2015) Uncovering Cooperative Behaviors with Sparse Historical Behavior Data in the Spatial Games. Applied Mathematics and Computation, 271, 317-322. https://doi.org/10.1016/j.amc.2015.09.023

[7] Szolnoki, A. and Perc, M. (2017) Evolutionary Dynamics of Cooperation in Neutral Populations. New Journal of Physics, 20, Article ID: 013031. https://doi.org/10.1088/1367-2630/aa9fd2

[8] Qin, J.H., Chen, Y.M., Fu, W.M., Kang, Y. and Perc, M. (2017) Neighborhood Diversity Promotes Cooperation in Social Dilemmas. IEEE Access, 6, 5003-5009. https://doi.org/10.1209/0295-5075/118/18002

[9] Wang, Z., Bauch, C.T., Bhattacharyya, S., D’Onofrio, A., Manfredi, P., Perc, M., Perra, N., Salathe, M. and Zhao, D.W. (2016) Statistical Physics of Vaccination. Physics Reports, 664, 1-113. https://doi.org/10.1016/j.physrep.2016.10.006

[10] D’Orsogna, M.R. and Perc, M. (2014) Statistical Physics of Crime: A Review. Physics of Life Reviews, 12, 1-21. https://doi.org/10.1016/j.plrev.2014.11.001

[11] Nash, J.F. (1950) Equilibrium Points in N-Person Games. Proceedings of the National Academy of Sciences, 36, 48-49. https://doi.org/10.1073/pnas.36.1.48

[12] Kaniovski, Y.M. and Young, H.P. (1994) Learning Dynamics in Games with Stochastic Perturbations. Games and Economic Behavior, 11, 330-363. https://doi.org/10.1006/game.1995.1054

[13] Conlisk, J. (1996) Why Bounded Rationality. Journal of Economic Literature, 34, 669-700.

[14] Smith, J.M. (1982) Evolution and the Theory of Games. Cambridge University Press, Cambridge. https://doi.org/10.1017/CBO9780511806292

[15] Nowak, M.A. (2006) Evolutionary Dynamics: Exploring the Equations of Life. Harvard University Press, Cambridge. https://doi.org/10.2307/j.ctvighw98

[16] Segal, N.L. and Hershberger, S.L. (1999) Cooperation and Competition between Twins: Findings from a Prisoner's Dilemma Game. Evolution and Human Behavior, 20, 29-51. https://doi.org/10.1016/S1090-5138(98)00039-7

[17] Cardillo, A., Petri, G., Nicosia, V., Sinatra, R., Gomez-Gardenes, J. and Latora, V. (2014) Evolutionary Dynamics of Time-Resolved Social Interactions. Physical Review E, 90, Article ID: 052825. https://doi.org/10.1103/PhysRevE.90.052825

[18] Axelrod, R. and Hamilton, W.D. (1981) The Evolution of Cooperation. Science, 211, 1390-1396. https://doi.org/10.1126/science.7466396

[19] Nowak, M.A. and May, R.M. (1992) Evolutionary Games and Spatial Chaos. Nature, 359, 826-829. https://doi.org/10.1038/359826a0

[20] Nowak, M.A. and May, R.M. (1993) The Spatial Dilemmas of Evolution. International Journal of Bifurcation and Chaos, 3, 35-78. https://doi.org/10.1142/S0218127493000040

[21] Bilancini, E., Boncinelli, L. and Wu, J.B. (2018) The Interplay of Cultural Intoler- 
ance and Action-Assortativity for the Emergence of Cooperation and Homophily. European Economic Review, 102, 1-18. https://doi.org/10.1016/j.euroecorev.2017.12.001

[22] Xu, H.D., Fan, S.H., Tian, C.Z. and Xiao, X.R. (2019) Effect of Strategy-Assortativity on Investor Sharing Games in the Market. Physica A, 514, 211-225.

https://doi.org/10.1016/j.physa.2018.09.056

[23] Szabó, G. and Fáth, G. (2007) Evolutionary Games on Graphs. Physics Reports, 446, 97-216. https://doi.org/10.1016/j.physrep.2007.04.004

[24] Chen, X.J., Fu, F. and Wang, L. (2008) Influence of Different Initial Distributions on Robust Cooperation in Scale-Free Networks: A Comparative Study. Physics Letters $A$, 372, 1161-1167. https://doi.org/10.1016/j.physleta.2007.09.044

[25] Langer, P., Nowak, M.A. and Hauert, C. (2008) Spatial Invasion of Cooperation. Journal of Theoretical Biology, 250, 634-641. https://doi.org/10.1016/j.jtbi.2007.11.002

[26] Masuda, N. (2012) Evolution of Cooperation Driven by Zealots. Scientific Reports, 2, Article No. 646. https://doi.org/10.1038/srep00646

[27] Yang, Z.H., Li, Z. and Wang, L. (2020) Evolution of Cooperation in a Conformity-Driven Evolving Dynamic Social Network. Applied Mathematics and Computation, 379, Article ID: 125251. https://doi.org/10.1016/j.amc.2020.125251

[28] Liu, Y.K., Zhang, L., Chen, X.J., Ren, L. and Wang, L. (2013) Cautious Strategy Update Promotes Cooperation in Spatial Prisoner's Dilemma Game. Physica A, 392, 3640-3647. https://doi.org/10.1016/j.physa.2013.04.031

[29] Li, Y., Lan, X., Deng, X.Y., Sadiq, R. and Deng, Y. (2014) Comprehensive Consideration of Strategy Updating Promotes Cooperation in the Prisoner's Dilemma Game. Physica A, 403, 284-292. https://doi.org/10.1016/j.physa.2014.02.051

[30] Poncela, J., Gomez-Gardenes, J., Floria, L.M. and Moreno, Y. (2007) Robustness of Cooperation in the Evolutionary Prisoner's Dilemma on Complex Networks. New Journal of Physics, 9, 281-289. https://doi.org/10.1088/1367-2630/9/6/184

[31] Flood, M.M. (1958) Some Experimental Games. Management Science, 5, 5-26. https://doi.org/10.1287/mnsc.5.1.5

[32] Colman, A.M. (1995) Game Theory and Its Applications in the Social and Biological Sciences. Routledge, London.

[33] Weibull, J.W. (1995) Evolutionary Game Theory. The MIT Press, Cambridge.

[34] Hauert, C. and Szabo, G. (2005) Game Theory and Physics. American Journal of Physics, 73, 405-414. https://doi.org/10.1119/1.1848514

[35] Guan, J.Y., Wu, Z.X., Huang, Z.G., Xu, X.J. and Wang, Y.H. (2006) Promotion of Cooperation Induced by Nonlinear Attractive Effect in Spatial Prisoner's Dilemma Game. Europhysics Letters, 76, 1214-1220. https://doi.org/10.1209/epl/i2006-10381-4 